\title{
Metal-Assisted and Solvent-Mediated Synthesis of Two-Dimensional Triazine Structures on Gram Scale
}

\author{
Abbas Faghani, Mohammad Fardin Gholami, Matthias Trunk, Johannes Müller, Pradip \\ Pachfule, Sarah Vogl, Ievgen Donskyi, Mingjun Li, Philip Nickl, Jingjing Shao, Michael \\ Huang, Wolfgang E. S. Unger, Raul Arenal, Christoph T. Koch, Beate Paulus, Jürgen P. \\ Rabe, Arne Thomas*, Rainer Haag and Mohsen Adeli*
}

\begin{abstract}
Covalent triazine frameworks are an emerging material class, which have shown promising performance for a range of applications. In this work, we report on a metalassisted and solvent-mediated reaction between calcium carbide and cyanuric chloride, as cheap and commercially available precursors, to synthesize two-dimensional triazine structures (2DTSs). The reaction between dimethylformamide and cyanuric chloride was promoted by calcium carbide and resulted in dimethylamino-s-triazine intermediates which in turn undergo nucleophilic substitutions. This reaction was directed into two dimensions by calcium ions derived from calcium carbide and induced the formation of 2DTSs. The role of calcium ions to direct the two-dimensionality of the final structure was simulated using DFT and further proven by synthesizing molecular intermediates. The water content of the reaction medium was found to be a crucial factor that affected the structure of the products dramatically. While 2DTSs were obtained at anhydrous conditions, a mixture of graphitic material/2DTSs or only graphitic material (GM) was obtained in aqueous solutions. Taking advantage of the straightforward and gram-scale synthesis of 2DTSs, as well as their photothermal and photodynamic properties, they are promising materials for a wide range of future applications including bacteria and virus incapacitation.
\end{abstract}

Keywords: Two-dimensional polymer, two-dimensional polymerization, calcium carbide, triazine framework 


\section{INTRODUCTION}

Reticular chemistry has notably extended the scope of two and three-dimensional porous structures. ${ }^{1-4}$ Two-dimensional polymers, ${ }^{5}$ metal organic frameworks, ${ }^{6}$ covalent organic frameworks, ${ }^{7}$ and polymeric networks ${ }^{8}$ with complex but known structures are designed and prepared by this strategy. These materials have shown a high potential for the future applications ranging from energy storage ${ }^{9}$ to membrane separation. ${ }^{10}$

However, two-dimensional covalent organic frameworks with versatile but defined structures are at the forefront of this rapidly developing field of chemistry. ${ }^{11-14}$ Also, triazinebased structures with unique physicochemical properties have been intensively investigated in the last decade. ${ }^{15-19}$ Microporous triazine-based structures ${ }^{20}$ with diverse applications in gas adsorption and storage, ${ }^{21-25}$ catalysis, ${ }^{26-29}$ and energy storage ${ }^{30-32}$ have been prepared by a variety of synthetic methods. ${ }^{33}$ Trimerization of nitriles, for example, is a straightforward and one-pot synthetic approach by which triazine-based structures with high surface area have been prepared. ${ }^{16}$ However, most of the current synthetic methods require high reaction temperatures, which lead to partial carbonization and undefined structures. ${ }^{34-35}$ Triazine-based structure $^{20}$ synthesized by such methods do not show an electronic band gap and useful optoelectronic properties, due to side reactions at high temperatures. ${ }^{35}$ Therefore, it is crucial to develop new strategies to synthesize more defined structures with useful optical and electronic properties. ${ }^{32}$ Recently, the synthesis of triazine-based structures has been improved using acid-catalyzed microwave ${ }^{36}$ and mechano-chemistry, ${ }^{37}$ which elaborate the current harsh synthetic conditions of ionothermal trimerization. However, the synthesis of triazine structures in solution, through which kinetic and thermodynamic parameters of reactions could be manipulated, has not been considerably developed. The lack of efficient synthetic methods in solution is a problem that has limited the structural diversity of triazine-based structures and hampered their practical usage. ${ }^{19}$ In addition to synthetic challenges, the preparation of triazine-based structures in mono- and few-layered forms is another problem for attaining unique characteristics of two-dimensional nanomaterials. ${ }^{28,}$ 38-39 This objection affects the efficiency of these materials, where thickness and number of layers play a significant role.

Recently, triazine-based structures have been synthesized by a polycondensation reaction of aldehydes and amidines at relatively mild conditions. ${ }^{15}, 35$ Although frameworks synthesized by this method have shown layered structures, superior to previous works, they could not be found in mono-layer form. ${ }^{39}$ Stacking of covalent triazine-based structures in multi-layered structures diminishes their accessible surface area and changes their 
physicochemical properties. Aldehyde-amidine polycondensation is a straightforward reaction for gram-scale synthesis of triazine-based structures but is limited by availability of the synthetic monomers. Furthermore, purification of triazine based structures is a timeconsuming process and requires washing at relatively high temperatures. ${ }^{40}$ This process is not compatible with reactive functional groups and less stable frameworks. Very recently, triazine-based olefin-linked structures ${ }^{41}$ have been reported, where triazine units can be stitched on to other organic linkers via covalent bonding. ${ }^{42}$ Although, these fully conjugated and crystalline triazine-based structures have shown interesting properties, this research topic is still in its infancy and need more attention for bringing out a general synthesis protocol for various structures in bulk-scale. Accordingly, scale-up, cost-efficiency, purification process, reaction parameters, and environmental aspects of classical synthetic methods require to be improved to economically obtain covalent mono-layer triazine frameworks with desired physicochemical properties.

In this work, we have addressed these challenges by metal-assisted and solventmediated synthesis of two-dimensional triazine structures (2DTSs). Reaction between calcium carbide and cyanuric chloride in dimethylformamide (DMF) resulted in 2DTSs with several micrometer lateral sizes. While cyanuric chloride acts as the source of triazine units, calcium carbide provides both acetylide linkages and calcium ions. The reaction was mediated by DMF and directed in two dimensions by calcium ions, leading to 2DTSs after trimerization of triple bonds. Adding water to the reaction medium changed the structure of the product from 2DTSs, in anhydrous conditions, to graphitic materials. Taking advantage of the $\pi$-conjugated system of their triazine backbone, 2DTSs can be developed for future applications such as photocatalysis, ${ }^{43}$ electrocatalysis, ${ }^{44}$ supercapacitors ${ }^{32}$ and Li-ion battery. ${ }^{45}$ However, in this manuscript, we have focused on the synthesis and physicochemical properties of 2DTSs, in addition to their mechanistic investigations.

\section{RESULTS AND DISCUSSION}

The reaction between cyanuric chloride and DMF at different conditions results in versatile intermediates. ${ }^{46-48}$ Herein, we demonstrate that this reaction, in the presence of calcium carbide, yields 2-(N,N-dimethylamino)-4,6-dichloro-s-triazine, which undergoes consecutive nucleophilic aromatic substitutions by acetylide dianions and produces two-dimensional triazine structures after trimerization (Figure 1). Calcium ions directed the carbon-carbon coupling of monomers in two dimensions and were eventually removed by washing (see page 
S7). This reaction was performed under different conditions and the effect of water content on the structure of the products was monitored (Figure 1).

In order to carry out reactions, calcium carbide and cyanuric chloride were mixed and stirred in bulk under inert atmosphere and then dispersed in DMF. Depending on the amount of water present during the reaction, the product varied from crystalline graphitic material to pure 2DTSs (Figure 1). The structures of the products were analyzed by different spectroscopy and microscopy methods as well as elemental and thermal analysis. Also, the reaction mechanisms were investigated by synthesizing different model compounds and studying the structure of intermediates (vide infra).

High resolution transmission electron microscopy (HRTEM) and scanning electron microscopy (SEM) images revealed the formation of layered and bulk structures with lateral sizes of several micrometers for the products of reactions at different conditions (Figures $2 \mathrm{a}, \mathrm{b}, \mathrm{d}, \mathrm{e})$. Further investigations revealed that water affected the structure of the reaction products dramatically. The product of the reaction between calcium carbide and water was crystalline with a measured in-plane lattice constant of the hexagonal crystal of approximately $0.248 \mathrm{~nm}$ (min: $0.241 \mathrm{~nm}$, max: $0.254 \mathrm{~nm}$, see Figure S1). This corresponds well to the inplane lattice constant of graphite $(\mathrm{a}=0.246 \mathrm{~nm})$. These results were consistently obtained by HRTEM imaging and electron diffraction in the SEM. Furthermore, EDX elemental analysis of the crystalline particles concluded approximately $99 \%$ carbon, which indicates, together with the hexagonal unit cell, that the material is graphite. In anhydrous conditions (see page S7), however, a product with an amorphous structure was obtained (Figure 2c, Figure S4), which we determined to be 2DTSs. Electron energy loss spectroscopy (EELS) spectra showed discrete peaks at $\sim 285 \mathrm{eV}$ and $\sim 400 \mathrm{eV}$, which were assigned to carbon and nitrogen in the backbone of this material (Figure S3). ${ }^{49-52}$ 
a

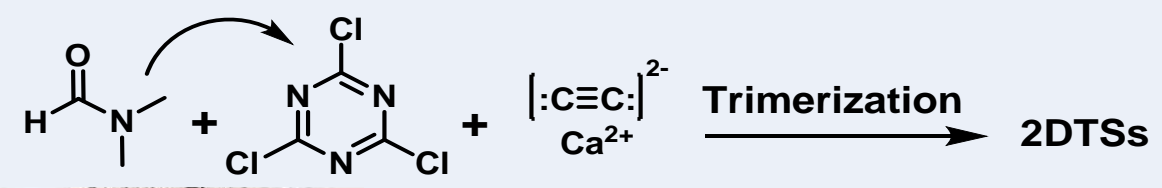

b

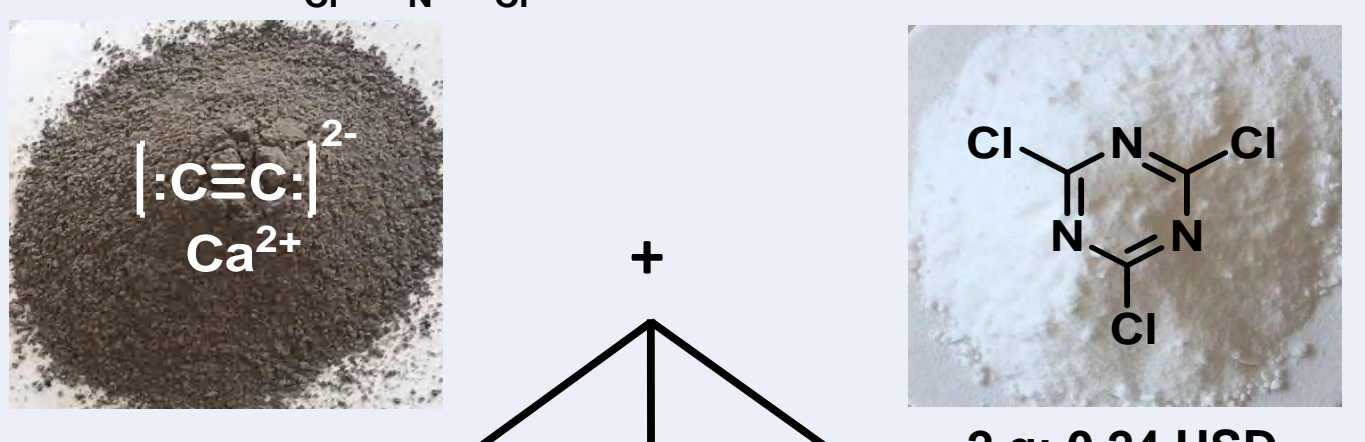

$2 \mathrm{~g} ; 0.28$ USD

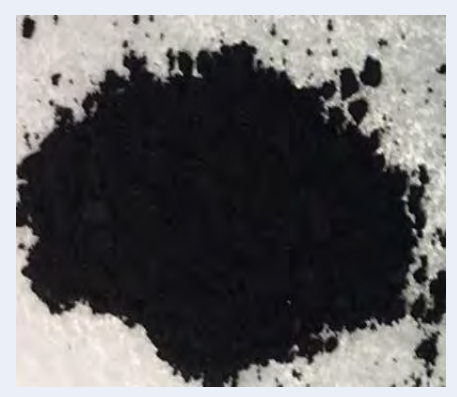

Graphitic

Material (GM)
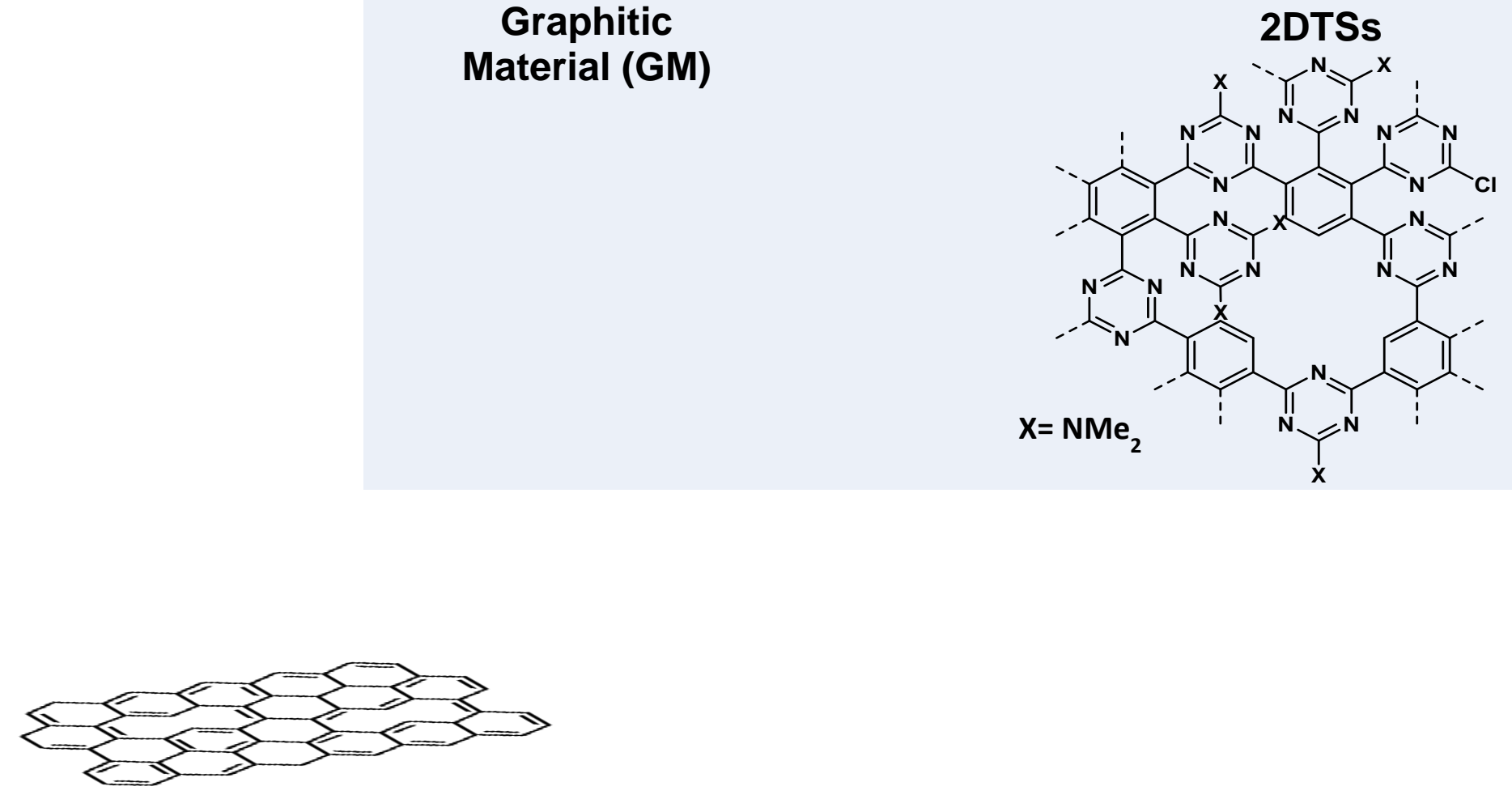

GM/2DTSs mixture

$2 \mathrm{~g} ; 0.24$ USD

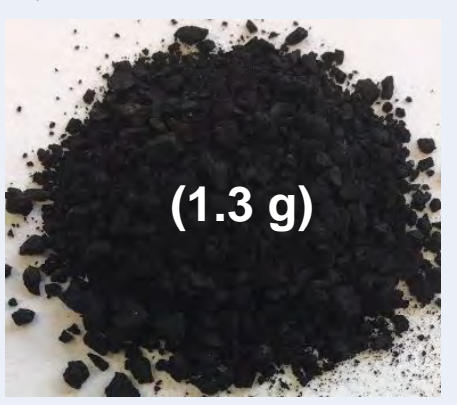

2DTSs

60

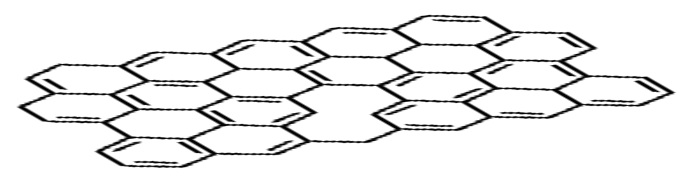

Figure 1. (a) Reaction between triazine and calcium carbide in anhydrous DMF resulted in 
2DTSs. (b) The product of the reaction between calcium carbide and triazine was strongly influenced by the water content of the solvent. While graphitic material (GM) was the main product in aqueous solutions (water, $25^{\circ} \mathrm{C}, 30$ minutes), 2DTSs were obtained in anhydrous DMF (120 ${ }^{\circ} \mathrm{C}, 72$ hours). Also, a mixture of GM/2DTSs was created in non-anhydrous DMF $\left(120^{\circ} \mathrm{C}, 72\right.$ hours). The cost-effectiveness of the reaction is shown by the estimated prices and quantities of the precursors. Prices of reagents were monitored from commercial suppliers (see page S3).

The nitrogen content of the reaction products at different conditions, which is an indicator for the triazine rings, was further investigated by elemental analysis. While the nitrogen content of the reaction product in water (GM) was close to zero, it increased to $\sim 21 \%$ for the product of reaction under anhydrous conditions (Table S1). Under anhydrous conditions the carbon/nitrogen ratio of the product was 2.6, yielding approximately a $\mathrm{C}_{3} \mathrm{~N}_{1}$ molecular formula for 2DTSs (Table S1). This carbon/nitrogen ratio indicated the formation of graphitic domains ${ }^{53-54}$ in 2DTSs by acetylide cross-coupling and trimerization (Figure 3d).

The topography and size of the product of reaction under anhydrous condition was further investigated by scanning force microscopy in quantitative nanomechanical mapping mode (SFM-QNM) on a freshly cleaved mica surface (Figures $2 \mathrm{~g}$, h and Figures S5a,b). It revealed layered structures with lateral sizes of 2 to 3 micrometers and thicknesses of 100 to $350 \mathrm{~nm}$, with layers and terraces including smaller layers with self-overlapping regions (folds) and an inhomogeneous distribution of mobile and small interconnected particles above the larger flakes, with a RMS roughness of $1 \mathrm{~nm}$. Since the heights of the layers were between 5 to $10 \mathrm{~nm}$, we believe that we did not observe a single-layer exfoliation of the layered structures. However, exfoliated multilayered structures exhibited self-avoiding behavior as evident in back folding, ${ }^{55-56}$ which may be simulated on macroscale using a sheet of paper for Figure 2h (Figure S5c). Self-avoidance of a structure is a direct consequence of its covalent bonding within a $2 \mathrm{D}$ network. This is indeed not the case for self-assembled 2D structures such as lipid bilayers/membranes, as those structures can self-penetrate and restructure back to a single layer rather than stabilize in a folded conformation. An observation of folding in case of $2 \mathrm{D}$ covalent structures is possible only when there is an overall gain in the energy of the system. This can be achieved by van der Waals interlayer forces (similar to those present within layers of a crystal) triggered by drying process during surface deposition. ${ }^{56}$ The product of the reaction between calcium carbide and cyanuric chloride in DMF under ambient condition (Figure 1, midline), which is called 2DTSs/GM, was also investigated by different 
characterization methods including TEM, EDX, elemental and thermal analysis. It was found that this product is always a mixture of 2DTSs and graphitic material (Table S1, Figure 3b). Attenuated total reflection infrared spectroscopy (ATR-FTIR) of 2DTSs revealed a broad absorbance band at $\sim 1394-1577 \mathrm{~cm}^{-1}$ that corresponds to carbon-carbon and carbon-nitrogen double bonds. The absence of the triple-bond absorbance and the appearance of $\mathrm{C}=\mathrm{C}$ vibrations in the IR spectrum of 2DTSs are attributed to the trimerization of triple bonds and the formation of benzene rings (Figure S6). Also, an absorbance band at $3300 \mathrm{~cm}^{-1}$ indicated presence of hydroxyl functional groups in the structure of 2DTSs, which have been created by the reaction between unreacted chlorine atoms of cyanuric chloride and water in the purification process.
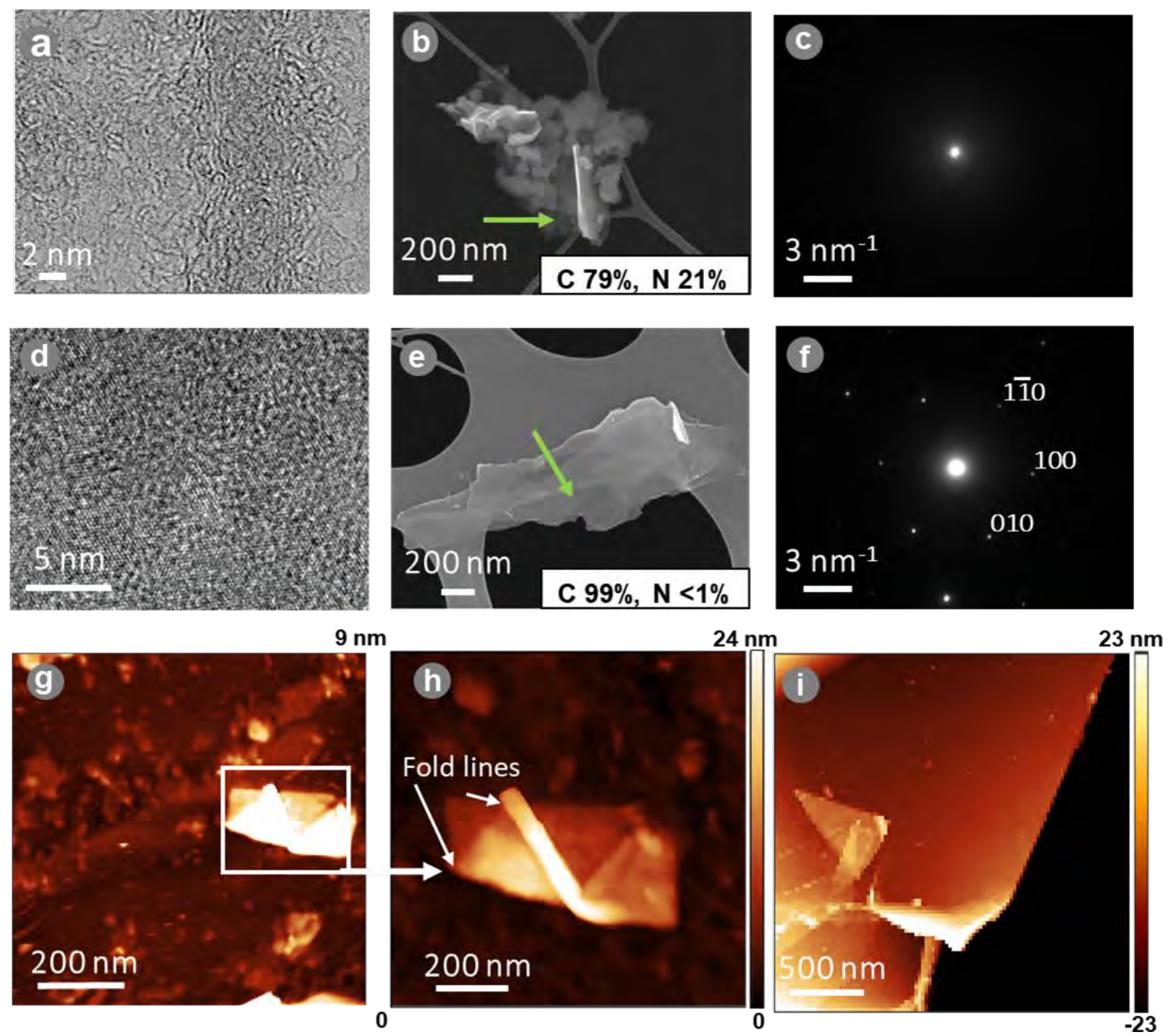

Figure 2. The products were investigated by different microscopy methods including HRTEM, SEM and SFM. (a) and (d) HRTEM images of 2DTSs and GM, respectively. (b) and (e) SEM images of 2DTSs and graphitic material on top of holey carbon TEM grids, respectively. (Insets are carbon and nitrogen contents of the indicated area obtained by energy-dispersive X-ray spectroscopy (EDX).) (c) and (f) Diffraction patterns of 2DTSs and 
GM at the indicated areas of (b) and (e) that show amorphous and crystalline characteristics for these materials, respectively. The in-plane unit cell parameter of the hexagonal lattice of GM was on average $0.248 \mathrm{~nm}$. (g) SFM-QNM height image of the product of reaction in anhydrous condition with plateaus and terraces (more images in Figure S5), (h) A close-up image of a sheet obtained in anhydrous condition with back folding. Planar layer topography is evident from the height image. (i) SFM-QNM height images of a large plateau of graphitic material.

${ }^{13} \mathrm{C}$ solid-state cross-polarization-magic-angle-spinning (CP-MAS) nuclear magnetic resonance (NMR) spectra of 2DTS showed a broad signal at 148-162 ppm, which is assigned to the carbon atoms of triazine rings ${ }^{57-58}$ and a signal at $27 \mathrm{ppm}$ for $-\mathrm{NMe}_{2}$ groups (Figure 3a). In this spectrum, a broad signal centered at $126 \mathrm{ppm}$ is assigned to benzene rings, which is a further proof for the trimerization of triple bonds (Figure $3 a$ ). 

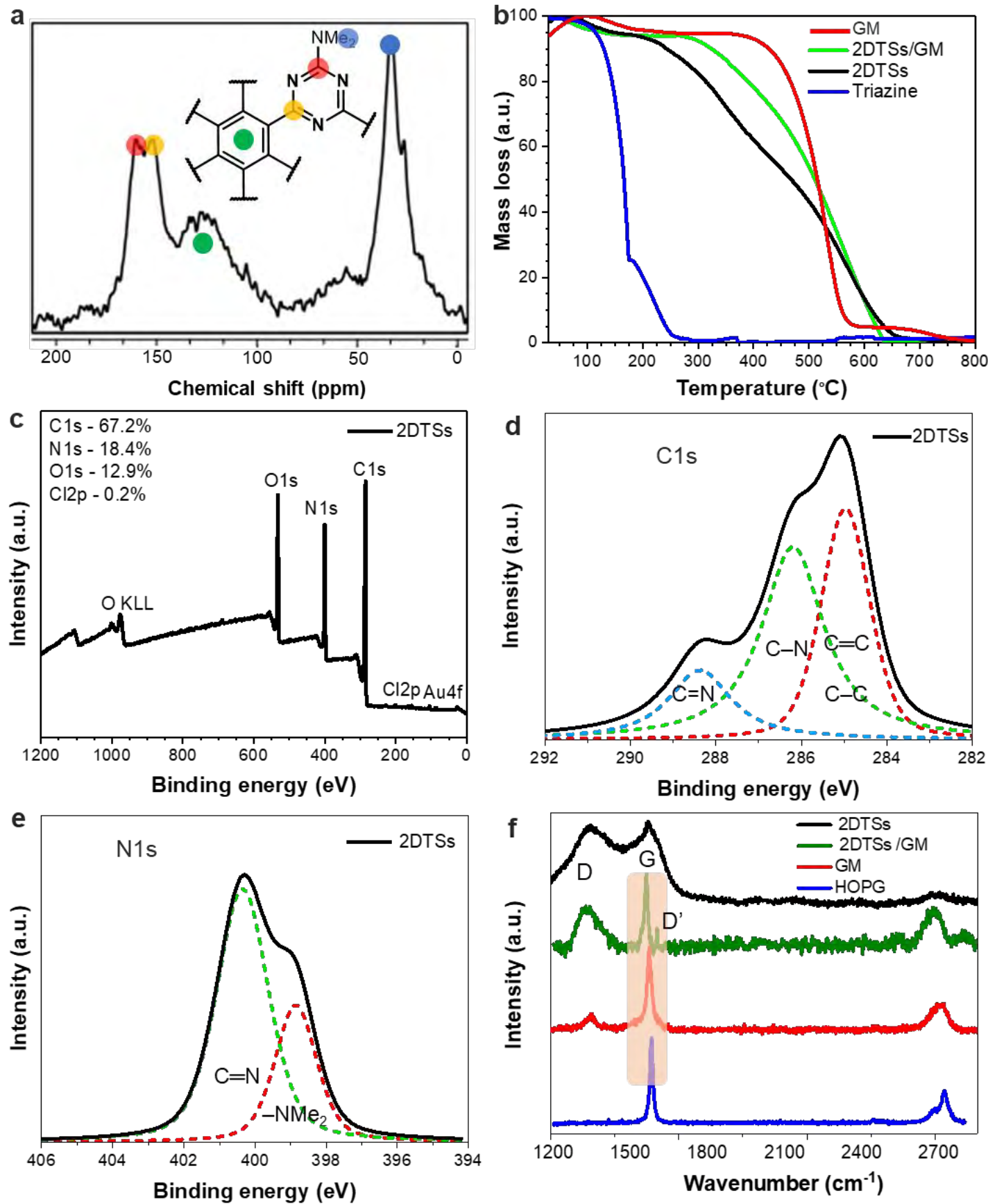

Figure 3. Characterization of 2DTSs by spectroscopy methods and thermal analysis. (a) ${ }^{13} \mathrm{C}$ CP-MAS-NMR spectrum of 2DTSs showed distinguished signals for the triazine and benzene rings as well as $-\mathrm{NMe}_{2}$ functional groups. (b) TGA thermograms of 2DTSs, cyanuric chloride GM, and GM/2DTSs. (c) Survey XPS spectrum of 2DTSs with quantification table of the main elements. Absence of the calcium peak after washing process confirmed the successful removal of metal ions. Au4f peak originates from the substrate. (d) Highly resolved XPS C1s spectrum and (e) highly resolved XPS N1s spectrum of 2DTSs. Fit parameters for each spectrum and interpretations are shown in table S2. (f) A close up of 
Raman spectra overlay of the G peak and D' peak regions of the 2DTSs, GM, and highly oriented pyrolytic graphite (HOPG).

The thermal behavior of 2DTSs was investigated by thermogravimetric analysis (TGA) in air. Their thermal stability correlated with the nitrogen content of the products. Materials with lower nitrogen contents, which are the products of reactions in aqueous solution or non-anhydrous condition, showed higher thermal stability. This is an indicator for the higher graphitic content of these materials. Based on literature reports, the reaction between water and calcium carbide is the source of graphitic materials in our reactions. ${ }^{53}$ While the production of GM occurs at room temperature, the reaction between calcium carbide and cyanuric chloride requires temperatures around $120^{\circ} \mathrm{C}$. Therefore, the production of GM is kinetically favored, and cyanuric chloride does not play a role at low temperatures. In the TGA thermogram of 2DTSs, weight losses in low and high temperature ranges are assigned to the detachment of $-\mathrm{NMe}_{2}$ functional groups and decomposition of the backbone of this nanomaterial, respectively (Figure 3b). The structure of 2DTSs was further investigated by X-ray photoelectron spectroscopy (XPS). Carbon, nitrogen, as the main components, and oxygen were detected in the survey spectrum of 2DTSs (Figure 3c). The highly resolved C1s XPS spectrum (Figure 3d) showed three intense C1s component peaks at $288.4 \mathrm{eV}$, representing carbon atoms of triazine rings, ${ }^{59}$ and at 286.2 and $285.0 \mathrm{eV}$, corresponding to the $-\mathrm{NMe}_{2}$ groups and carbons of benzene rings, respectively. ${ }^{54,58,60}$ Two components in the N1s peak at $400.4 \mathrm{eV}$ and $398.9 \mathrm{eV}$ were assigned to the nitrogen atoms of triazine rings and $\mathrm{NMe}_{2}$ groups, respectively (Figure 3e). ${ }^{58,61}$ The absence of the calcium peak in the survey spectrum of 2DTSs is a proof of the successful removal of calcium upon purification (Figure $3 \mathrm{c})$.

In order to complement the characterization of the synthesized materials through investigation of their polymorphism and crystallinity, their Raman spectra were individually evaluated and mapped (Figures $3 \mathrm{f}$ and S7). The G peaks of 2DTSs and GM were appeared at $1562 \mathrm{~cm}^{-1}$ and $1582 \mathrm{~cm}^{-1}$, respectively. In the case of 2DTSs, broadening of Raman peaks at $1320-1360 \mathrm{~cm}^{-1}$ area, centered at $1345 \mathrm{~cm}^{-1}$, as well as peaks at $1550-1620 \mathrm{~cm}^{-1}$ area with a pronounced peak at 1562 to $1565 \mathrm{~cm}^{-1}$ was observed. As it is previously reported by Ferrari et al., ${ }^{62}$ the $\mathrm{C}=\mathrm{N}$ vibrational frequencies are very similar to the $\mathrm{C}=\mathrm{C}$ within the 1000 to $2000 \mathrm{~cm}^{-}$ ${ }^{1}$ range. Therefore, the observed peaks in the Raman spectra of 2DTSs were assigned to a structure with the embedded nitrogen atoms. Furthermore, increasing the nitrogen content of products affected the $\mathrm{G}$ peak position in their Raman spectra, when visible laser light was 
used. For example, for a product with 20-30\% nitrogen content, a $\mathrm{G}$ peak at $1560-1565 \mathrm{~cm}^{-1}$ was observed which is in agreement with the reported data in literature. ${ }^{62}$ This downshift in comparison with the G peak of HOPG was assigned to the difference between GM composition and HOPG. ${ }^{62}$ The direct correlation between this shift and the nitrogen content of the materials is a further proof for the preparation of 2DTSs at anhydrous conditions. Furthermore, 2DTSs/GM mixtures exhibit Raman bands at $\sim 1332 \mathrm{~cm}^{-1}$ and $\sim 1564 \mathrm{~cm}^{-1}$ with an occasional weak and broad band at $\sim 1610-1620 \mathrm{~cm}^{-1}$ in addition to a broad band centered at $\sim 2700 \mathrm{~cm}^{-1}$ (Figure 3f). The Raman bands at $\sim 1345 \mathrm{~cm}^{-1}$ and $\sim 1620 \mathrm{~cm}^{-1}$, which correspond to the $\mathrm{D}$ peak, are assigned to the domain boundaries or nucleation sites within the structure. On the other hand, the D' peak observed occasionally at $\sim 1620 \mathrm{~cm}^{-1}$ demonstrates the doping by a hetero atom, suggesting nitrogen atoms of triazine in the structure of 2DTSs (Figure 3f). It is important to note that no band at $\sim 2100 \mathrm{~cm}^{-1}$ was observed in the spectrum of 2DTSs. In agreement with IR and NMR spectra, this is a further proof of the trimerization of triple bonds. ${ }^{63-64}$

After analyzing the chemical structure of the two-dimensional structures, the mechanism of their synthetic pathway and structures of intermediates were investigated. To avoid polymerization and to constrain the reaction to the molecular level, two active sites of cyanuric chloride were blocked by phenol, and 2-chloro-4,6-diphenoxy-1,3,5-s-triazine (1) was synthesized according to reported methods in literature ${ }^{65}$ (see Figures S8-11 for NMR and mass spectra). Then the reaction between 2-chloro-4,6-diphenoxy-1,3,5-s-triazine and calcium carbide was carried out in anhydrous DMF at $120{ }^{\circ} \mathrm{C}$. Different characterization methods showed that the chlorine atom of 2-chloro-4,6-diphenoxy-1,3,5-s-triazine was substituted by DMF leading to 2-(N,N-dimethylamino)-4,6-diphenoxy-s-triazine (2) (Figure 4a). The structure of compound (2) was investigated by single X-ray crystallography and spectroscopy methods (Figure 4b-d). ${ }^{1} \mathrm{H}-\mathrm{NMR}$ showed a chemical shift for the phenoxy protons of compound (1) after reaction with DMF. Also, a signal for $-\mathrm{NMe}_{2}$ protons appeared in the spectrum of compound (2) (Figure 4c). Comparison of ${ }^{13} \mathrm{C}$ NMR spectra of compounds (1) and (2) showed a distinct $\mathrm{C}-\mathrm{Cl}$ chemical shift from $173.8 \mathrm{ppm}$ to $167.4 \mathrm{ppm}$ after reaction with DMF. These results were in agreement with literature ${ }^{66-67}$ and confirmed substitution of the chlorine atom of compound (1) by $-\mathrm{NMe}_{2}$ groups, which were derived from DMF (Figure $4 \mathrm{~d})$. The structure of intermediate (2) was further proven by mass spectrometry. The signals at 309.1 and $331.1 \mathrm{~m} / \mathrm{z}$ are related to the molar mass of this compound accompanied by proton and sodium ion, respectively (calculated for $\mathrm{C}_{17} \mathrm{H}_{16} \mathrm{~N}_{4} \mathrm{O}_{2}[\mathrm{M}+\mathrm{H}]$ and $[\mathrm{M}+\mathrm{Na}]$ : 309.1 and 331.1, respectively, Figure S11). Accordingly, the signal of methyl groups in the CP-MAS- 
NMR of 2DTSs can be assigned to $-\mathrm{NMe}_{2}$ groups that are created by reaction between DMF and cyanuric chloride. Reaction between compound (2) and calcium carbide resulted in compounds (3 and $\mathbf{4}$ ). This reaction was monitored at intervals of two hours by MatrixAssisted Laser Desorption/Ionization Time-of-Flight (MALDI-TOF) and electrospray ionization mass spectrometry (ESI) for three days. Signal at $328.11 \mathrm{~m} / \mathrm{z}$ and $655.25 \mathrm{~m} / \mathrm{z}$ (Figures S12 and S13) are assigned to compounds $\mathbf{3}$ and 4, which were converted to compounds $\mathbf{5}$ and $\mathbf{6}$, respectively upon trimerization. Compound $\mathbf{4}$ was accompanied by one or two calcium ions (Figures S13 and S14), which showed that metal-ligand interactions were possible driving forces to inhibit rotation of triazine rings around linkages and direct polymerization in two dimensions. However, compound $\mathbf{4}$, which was accompanied by one calcium ion, could be more reactive toward trimerization, due to less steric hindrance. Compounds 5 and $\mathbf{6}$ were detected by MALDI-TOF (Figure 4e). These compounds proved formation of benzene rings in the structure of 2DTSs upon trimerization of triple bonds. Furthermore, fragments at $336 \mathrm{~m} / \mathrm{z}$ and $639 \mathrm{~m} / \mathrm{z}$ in the ESI mass spectrum of this model reaction indicated building blocks consisting benzene rings with six substituents (Figures S14 and S15). This result showed that calcium carbide can reacted with triazine rings from both sides because trimerization of the product of such a reaction results in a $2 \mathrm{D}$ polymer consisting of benzene rings with six substituents. It is worth noting that in compound 6 triazine rings were able to rotate to minimize the repulsion of electron pair of adjacent nitrogen atoms. However, in 2DTSs rotation of such units is restricted leading to an energetically unfavorable structure. Therefore, the units created by di-substituted calcium carbide can be mostly at the edges or defect sites, where the electron repulsion of adjacent nitrogen atoms is reduced. The backbone of 2DTSs consist mostly of graphitic domains or regions created by trimerization of mono-substituted calcium carbide or a mixture with its disubstituted analog (Figure 1b). In addition to MALDI-TOF spectra, ${ }^{1} \mathrm{H}$ NMR indicated the formation of mono-substituted calcium carbide. ${ }^{1} \mathrm{H}$ NMR of the product of reaction between 2-chloro-4,6-diphenoxy-1,3,5-s-triazine and calcium carbide showed clear signals for compounds $\mathbf{5}$, which could be created by trimerization of mono-substituted calcium carbide (Figure S16). The reaction mechanism was further investigated by synthesizing 2,4-dichloro6-phenoxy-1,3,5-s-triazine and polymerization of this monomer in DMF under the same reaction conditions (Scheme S1). In the MALDI-TOF spectrum of the product of this reaction, distinguished peaks at $243 \mathrm{n} \mathrm{m} / \mathrm{z}$ intervals, where $\mathrm{n}$ is a natural number, indicated a repeating unit with benzene substitutes (Figure S17). This repeating unit is a further proof of the nucleophilic substitution of chlorine atoms of triazine followed by trimerization. The role 
of DMF in the reaction between cyanuric chloride and calcium carbide was further proven by changing the solvent to N,N-dimethylacetamide toluene and mesitylene. Accomplishment of reaction in these solvents did not result in any detectable product. This experiment proved the critical role of DMF in the production of two-dimensional triazine structure. As indicated by compound (3), complexation of intermediates with calcium ions hampered the rotation of triazine rings around acetylide linkages and directed the consequent reactions in two dimensions (Figure S18). The directing role of calcium ions was further investigated by DFT functional in GPAW program ${ }^{68-70}$ (see page S23 and S24).

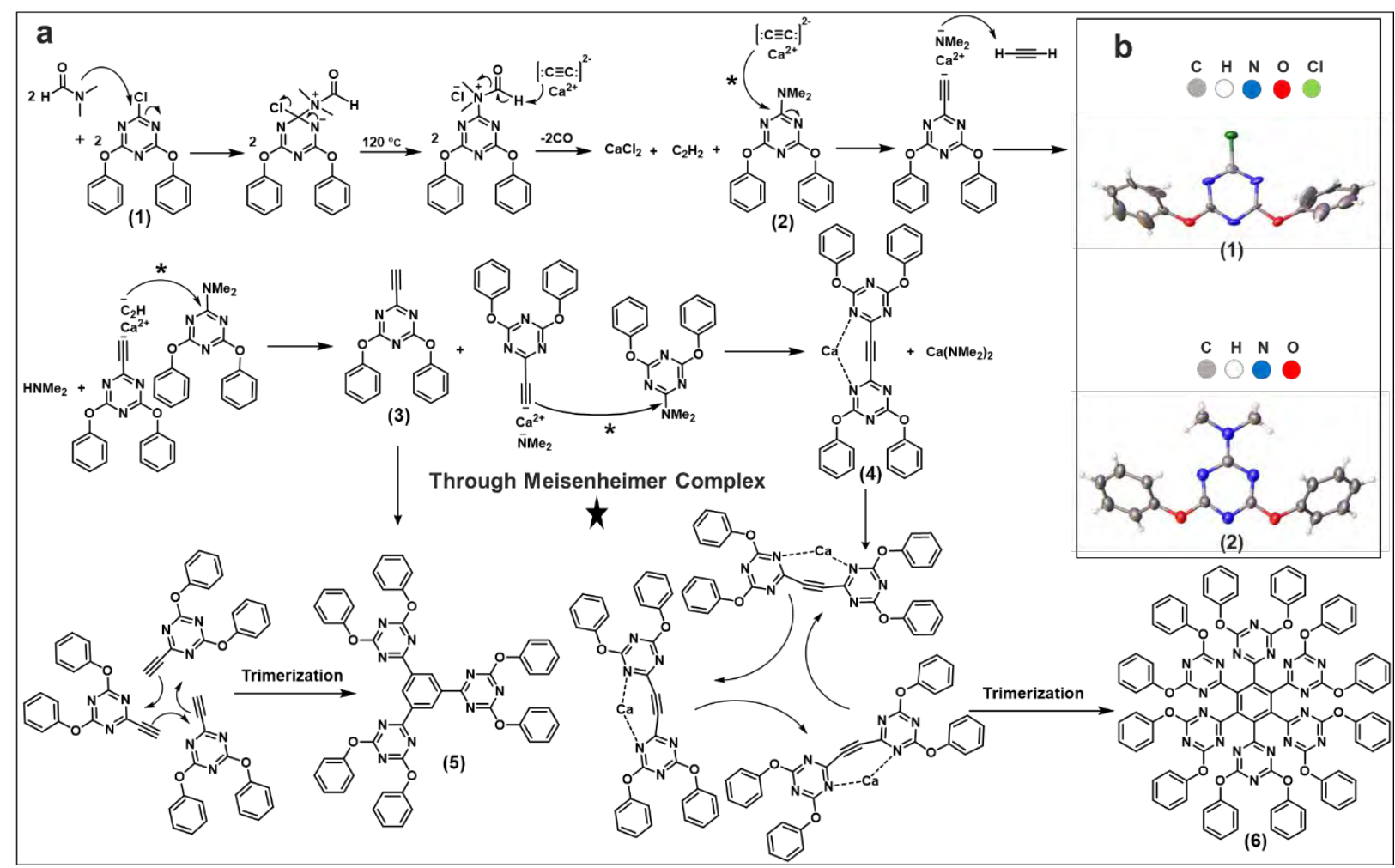



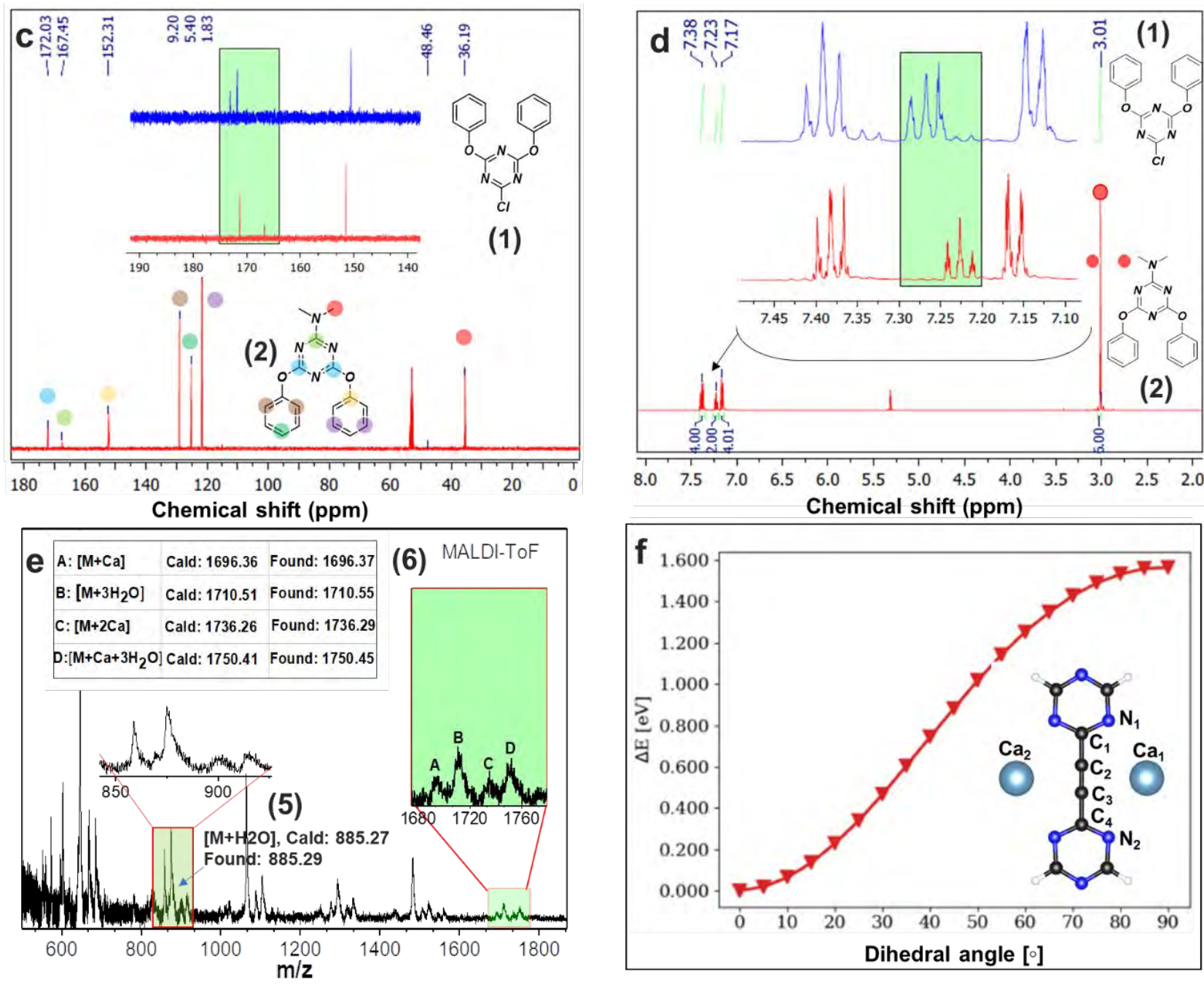

Figure 4. Investigation of the mechanism of the reaction between cyanuric chloride and calcium carbide in DMF using model compounds. (a) Reaction between compound (1) and DMF resulted in intermediate (2). Consequently, nucleophilic reaction between compound (2) and calcium carbide resulted in compounds $\mathbf{3}$ and $\mathbf{4}$ that converted to compounds $\mathbf{5}$ and $\mathbf{6}$ upon trimerization. (b) Single crystal XRD structure of compounds (1) and (2). (c) and (d) ${ }^{1} \mathrm{H}$ - and ${ }^{13} \mathrm{C}-\mathrm{NMR}$ spectra of compound (1) and compound (2), respectively. (e) Expanded MALDI-TOF spectrum of mixture of reaction between compound $\mathbf{1}$ and cyanuric chloride in DMF. (f) The simulated structure of model compound coordinated with two calcium ions (on both sides) and the total energy of the system with different dihedral angle with step size of $5^{\circ}$ (see details in Figure S18 and Table S3).

Coordination of one or two calcium ions with nitrogen atoms of the triazine rings in compound 4 (phenyl groups were replaced by hydrogen) caused $0.05 \mathrm{eV}$ and $1.6 \mathrm{eV}$ barrier energy, respectively, for rotation around $\mathrm{C}_{1}-\mathrm{C}_{2}$ and $\mathrm{C}_{3}-\mathrm{C}_{4}$ and bonds (Figure $4 \mathrm{f}$ ). These energy barriers are driving forces for growing triazine-benzene structures in two dimensions. 
According to the literature, calcium ions are able to form complexes with nitrogen containing ligands such as 1,10-phenanthroline, 4,4'-bipyridine and 2,2'-bipyridine. ${ }^{71-72}$ While interactions between these ligands and calcium ions are not as strong as oxygen containing ligands, ${ }^{73-74}$ they are able to induce enough rigidity for the two-dimensional coupling of monomers. ${ }^{68-70}$ The weakness of the interactions on the other hand enables that the metal ions can be removed completely from the final product. Another proof of the directing role of calcium ions was achieved by adding sodium carbonate to the reaction medium. When the reaction between cyanuric chloride and calcium carbide was performed in DMF and in the presence of sodium carbonate, no sheet-like structure was achieved, due to the production of sodium carbonate and absence of any driving force to conjugate monomers in a sheet-like structure (Figure S19).
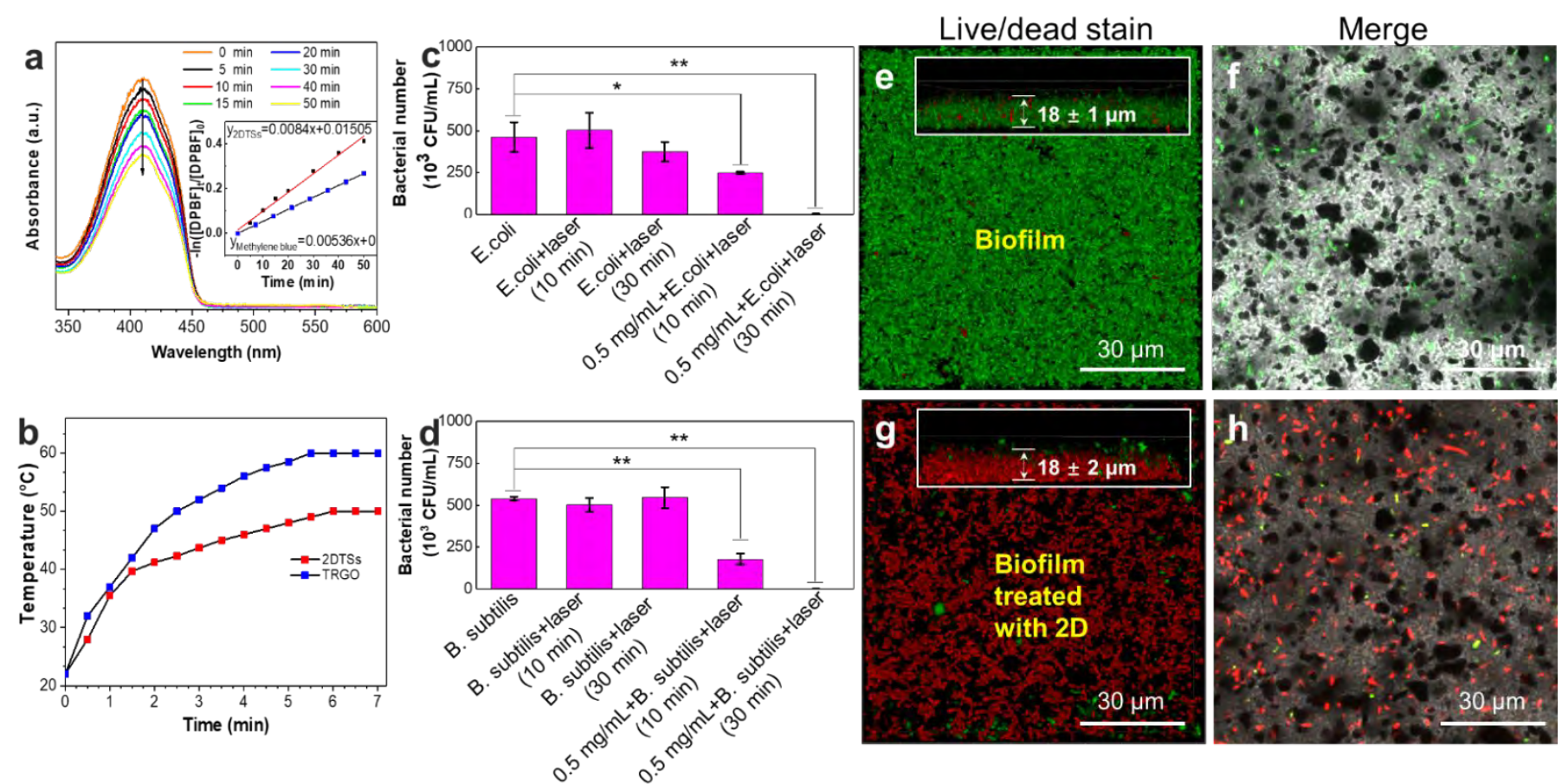

Figure 5. (a) Singlet oxygen generation kinetics of water dispersion of 2DTSs and (b) photothermal properties of 2DTSs under laser irradiation $\left(808 \mathrm{~nm}, 0.5 \mathrm{~W} / \mathrm{cm}^{2}\right.$ ). Thermally reduced graphene oxide (TRGO) was used as control, the concentration was $0.5 \mathrm{mg} / \mathrm{mL}$ in water (more details can be found in page S27). (c) and (d) antibacterial activities of samples $(0.5 \mathrm{mg} / \mathrm{mL})$ against E. coli and B. subtilis. E. coli and B. subtilis in PBS were used as controls. Data are presented as mean $\pm \mathrm{SD}, \mathrm{n}=3$. Statistically significant differences are indicated by $* \mathrm{p}<0.05$ and $* * \mathrm{p}<0.01$ compared with the control. (e-h) biofilm eradication test: the bacteria entrapped in biofilm were destroyed by 2DTSs upon laser irradiation. In 
live/dead assay, live and dead bacteria are shown by green and red colors respectively (details are available in page $S 27)$.

The photochemical properties of 2DTSs were investigated by different methods. 2DTSs showed a maximum UV absorption at 235-276 nm corresponding to $\mathrm{n} \rightarrow \pi^{*}$ transitions (Figure S20). Due to their large $\pi$-conjugated system, 2DTSs were able to produce singlet oxygen under irradiation with near infrared (NIR) laser $(808 \mathrm{~nm})$. This was confirmed using 1,3-Diphenylisobenzofuran (DPBF) as a specific singlet oxygen scavenger (Figure 5a). 2DTSs demonstrated a higher singlet oxygen production rate as observed by absorbance change of DPBF in comparison to methylene blue as a photosensitizer reference in similar solvent. This property could be used for different applications ranging from photodynamic therapy to photocatalyst applications. Laser irradiation of a water dispersion of 2DTSs elevated the medium temperature from $20{ }^{\circ} \mathrm{C}$ to $40{ }^{\circ} \mathrm{C}$ in 2 minutes (Figure $5 \mathrm{~b}$ ). The production of heat is less efficient than by thermally reduced graphene oxide but still in a suitable range for some applications including photothermal therapy or antibacterial activity. Accordingly, the antibacterial activity of 2DTSs under laser irradiation was investigated. While laser irradiation did not show a significant adverse effect against bacteria, 2DTSs $(0.5$ $\mathrm{mg} / \mathrm{mL}$ ) followed by 10 minutes laser irradiation resulted in incapacitation of $46 \%$ and $67 \%$ E.coli and B. subtilis respectively (Figures 5c,d). Prolongation of the laser irradiation to 30 min resulted in destruction of both gram positive and gram negative bacteria completely (in the detection limit). The bacteria entrapped in biofilm are more resistant to biocide, due to protection and sufficient nutrition inside the biofilm. Therefore, antibiofilm property of an antibacterial agent is of high relevance and determines its efficiency for the treatment of contaminated surfaces. Incubation of 2DTSs with an $18 \mu \mathrm{m}$ thick biofilm for $1 \mathrm{~h}$ followed by laser irradiation for $30 \mathrm{~min}$ resulted in destruction of bacteria inside the biofilm, proven by red fluorescence (Figures 5e, g). As Figures 5f and 5h show, 2DTSs with black color penetrated into the biofilm and incapacitated bacteria (more details in page S27).

Our results showed that the reaction between calcium carbide and triazine in DMF is an efficient strategy to develop versatile two-dimensional triazine structures through the concepts of reticular chemistry. Deep understanding of the mechanism of this reaction can help us to manipulate the structure of the products by changing reaction parameters including precursors, metal ion, solvent, and water content. 


\section{CONCLUSION}

The reaction between cyanuric chloride and calcium carbide under anhydrous conditions was mediated by $N, N$-dimethylformamide to produce $2 \mathrm{D}$ triazine-benzene structures on the gram scale. A mechanistic study revealed a dual role of the calcium carbide, first as a source of acetylide ions, which created benzene rings upon trimerization, and second the calcium ions could act as a two-dimensional directing force upon interaction with triazine rings. Adding water showed a high impact on the reaction route and in the presence of water, graphitic material was created. Taking advantage of its interesting physicochemical properties, straightforward synthesis and cheap precursors, the presented two-dimensional nanomaterial is a promising candidate for a wide range of future applications including photodynamic and photothermal therapy.

\section{ASSOCIATED CONTENT}

\section{Supporting Information}

Experimental details; SEM, TEM, HRTEM and SFM images; EELS spectra and PXRD diffractograms; IR, Raman \& NMR spectra; Mass spectrometry; XPS interpretation; computational analysis (DFT calculation).

\section{AUTHOR INFORMATION}

\section{Corresponding Authors}

Arne Thomas - Department of Chemistry / Functional Materials, Technische Universität Berlin, Hardenbergstraße 40, 10623 Berlin, Germany; Email: arne.thomas@tu-berlin.de

Mohsen Adeli - Institut für Chemie und Biochemie, Freie Universität Berlin, Arnimallee 22, Berlin 14195, Germany. Faculty of Science, Department of Chemistry, Lorestan University, Khorramabad, Iran; Email: m.aadeli@fu-berlin.de

\section{Authors}

Abbas Faghani - Institut für Chemie und Biochemie, Freie Universität Berlin, Arnimallee 22, Berlin 14195, Germany

Mohammad Fardin Gholami - Department of Physics \& IRIS Adlershof, HumboldtUniversität zu Berlin, Newtonstr. 15, 12489 Berlin Germany

Matthias Trunk - Department of Chemistry / Functional Materials, Technische Universität Berlin, Hardenbergstraße 40, 10623 Berlin, Germany

Johannes Müller - Department of Physics \& IRIS Adlershof, Humboldt-Universität zu Berlin, Newtonstr. 15, 12489 Berlin Germany 
Pradip Pachfule - Department of Chemistry / Functional Materials, Technische Universität Berlin, Hardenbergstraße 40, 10623 Berlin, Germany

Sarah Vogl - Department of Chemistry / Functional Materials, Technische Universität Berlin, Hardenbergstraße 40, 10623 Berlin, Germany

Ievgen Donskyi - Institut für Chemie und Biochemie, Freie Universität Berlin, Arnimallee 22, Berlin 14195, Germany. BAM - Federal Institute for Material Science and Testing, Division of Surface Analysis and Interfacial Chemistry, Unter den Eichen 44-46, 12205 Berlin, Germany

Mingjun Li - Institut für Chemie und Biochemie, Freie Universität Berlin, Arnimallee 22, Berlin 14195, Germany. Center for Health Science and Engineering, Tianjin Key Laboratory of Materials Laminating Fabrication and Interface Control Technology, School of Materials Science and Engineering, Hebei University of Technology, Tianjin 300130, China

Philip Nickl - Institut für Chemie und Biochemie, Freie Universität Berlin, Arnimallee 22, Berlin 14195, Germany. BAM - Federal Institute for Material Science and Testing, Division of Surface Analysis and Interfacial Chemistry, Unter den Eichen 44-46, 12205 Berlin, Germany

Jingjing Shao - Institut für Chemie und Biochemie, Freie Universität Berlin, Arnimallee 22, Berlin 14195, Germany

Michael Huang - Department of Physics \& IRIS Adlershof, Humboldt-Universität zu Berlin, Newtonstr. 15, 12489 Berlin Germany

Wolfgang E. S. Unger - BAM - Federal Institute for Material Science and Testing, Division of Surface Analysis and Interfacial Chemistry, Unter den Eichen 44-46, 12205 Berlin, Germany

Raul Arenal - Laboratorio de Microscopias Avanzadas (LMA), Instituto de Nanociencia de Aragon, Universidad de Zaragoza, 50018 Zaragoza, Spain. Fundacion ARAID, 50018 Zaragoza, Spain. Instituto de Ciencias de Materiales de Aragon, CSIC-Universidad de Zaragoza, 50009 Zaragoza, Spain

Christoph T. Koch - Department of Physics \& IRIS Adlershof, Humboldt-Universität zu Berlin, Newtonstr. 15, 12489 Berlin Germany

Beate Paulus - Institut für Chemie und Biochemie, Freie Universität Berlin, Arnimallee 22, Berlin 14195, Germany

Jürgen P. Rabe - Department of Physics \& IRIS Adlershof, Humboldt-Universität zu Berlin, Newtonstr. 15, 12489 Berlin Germany 
Rainer Haag - Institut für Chemie und Biochemie, Freie Universität Berlin, Arnimallee 22, Berlin 14195, Germany

\section{ACKNOWLEDGMENTS}

We thank the German Science Foundation (DFG) for financial support within the grants SFB 765 and SFB 658. M.F.G. and J.P.R. also acknowledge the support of the Cluster of Excellence "Matters of Activity. Image Space Material" funded by the DFG under Germany's Excellence Strategy EXC 2025. Furthermore, A.T. acknowledges the DFG for funding within the project TH 1463/12-1. We would like to thank Dr. Andreas Schäfer and Maiko Schulze for solid-NMR experiments as well as we appreciate the effort of Vahid Ahmadi Soureshjani for MALDI-TOF experiments. We would like to acknowledge M. Eng. Jörg M. Stockmann for operating the XPS instrument at the BAM, Prof. Stephanie Reich and Dr. Antonio Setaro for fruitful discussions. 2DTs-HRTEM and -EELS studies were conducted at the Laboratorio de Microscopias Avanzadas, Instituto de Nanociencia de Aragon, Universidad de Zaragoza, Spain. R.A. gratefully acknowledges the support from the Spanish Ministry of Economy and Competitiveness (MINECO) through project grant MAT2016-79776-P (AEI/FEDER, UE) and from the European Union H2020 programs ETN projects "Graphene Flagship" (785219) and "ESTEEM3" (823717).

\section{REFERENCES}

1. Pei, W.-Y.; Xu, G.; Yang, J.; Wu, H.; Chen, B.; Zhou, W.; Ma, J.-F., Versatile Assembly of MetalCoordinated Calix[4]resorcinarene Cavitands and Cages through Ancillary Linker Tuning. J. Am. Chem. Soc. 2017, 139 (22), 7648-7656.

2. Ma, T.; Kapustin, E. A.; Yin, S. X.; Liang, L.; Zhou, Z.; Niu, J.; Li, L.-H.; Wang, Y.; Su, J.; Li, J.; Wang, X.; Wang, W. D.; Wang, W.; Sun, J.; Yaghi, O. M., Single-crystal x-ray diffraction structures of covalent organic frameworks. Science 2018, 361 (6397), 48-52.

3. Diercks, C. S.; Liu, Y.; Cordova, K. E.; Yaghi, O. M., The role of reticular chemistry in the design of $\mathrm{CO} 2$ reduction catalysts. Nat. Mater. 2018, 17 (4), 301-307.

4. $\quad$ Feng, L.; Wang, K.-Y.; Lv, X.-L.; Yan, T.-H.; Li, J.-R.; Zhou, H.-C., Modular Total Synthesis in Reticular Chemistry. J. Am. Chem. Soc. 2020, 142 (6), 3069-3076.

5. Payamyar, P.; King, B. T.; Öttinger, H. C.; Schlüter, A. D., Two-dimensional polymers: concepts and perspectives. Chem Comm 2016, 52 (1), 18-34.

6. Baumann, A. E.; Burns, D. A.; Liu, B.; Thoi, V. S., Metal-organic framework functionalization and design strategies for advanced electrochemical energy storage devices. Commun. Chem. 2019, 2 (1), 86 .

7. Geng, K.; He, T.; Liu, R.; Dalapati, S.; Tan, K. T.; Li, Z.; Tao, S.; Gong, Y.; Jiang, Q.; Jiang, D., Covalent Organic Frameworks: Design, Synthesis, and Functions. Chem. Rev. 2020.

8. Molina, M.; Asadian-Birjand, M.; Balach, J.; Bergueiro, J.; Miceli, E.; Calderón, M., Stimuliresponsive nanogel composites and their application in nanomedicine. Chem. Soc. Rev. 2015, 44 (17), 6161-6186. 
9. Xu, F.; Yang, S.; Chen, X.; Liu, Q.; Li, H.; Wang, H.; Wei, B.; Jiang, D., Energy-storage covalent organic frameworks: improving performance via engineering polysulfide chains on walls. Chem. Sci. 2019, 10 (23), 6001-6006.

10. Yuan, S.; Li, X.; Zhu, J.; Zhang, G.; Van Puyvelde, P.; Van der Bruggen, B., Covalent organic frameworks for membrane separation. Chem. Soc. Rev. 2019, 48 (10), 2665-2681.

11. Côté, A. P.; Benin, A. I.; Ockwig, N. W.; O'Keeffe, M.; Matzger, A. J.; Yaghi, O. M., Porous, Crystalline, Covalent Organic Frameworks. Science 2005, 310 (5751), 1166-1170.

12. Montoro, C.; Rodríguez-San-Miguel, D.; Polo, E.; Escudero-Cid, R.; Ruiz-González, M. L.; Navarro, J. A. R.; Ocón, P.; Zamora, F., lonic Conductivity and Potential Application for Fuel Cell of a Modified Imine-Based Covalent Organic Framework. J. Am. Chem. Soc. 2017, 139 (29), 10079-10086.

13. Zhang, J.; Han, X.; Wu, X.; Liu, Y.; Cui, Y., Multivariate Chiral Covalent Organic Frameworks with Controlled Crystallinity and Stability for Asymmetric Catalysis. J. Am. Chem. Soc. 2017, 139 (24), 8277-8285.

14. Evans, A. M.; Parent, L. R.; Flanders, N. C.; Bisbey, R. P.; Vitaku, E.; Kirschner, M. S.; Schaller, R. D.; Chen, L. X.; Gianneschi, N. C.; Dichtel, W. R., Seeded growth of single-crystal two-dimensional covalent organic frameworks. Science 2018, 361 (6397), 52-57.

15. Liu, M.; Huang, Q.; Wang, S.; Li, Z.; Li, B.; Jin, S.; Tan, B., Crystalline Covalent Triazine Frameworks by In Situ Oxidation of Alcohols to Aldehyde Monomers. Angew. Chem. Int. Ed. 2018, 57 (37), 11968-11972.

16. Kuhn, P.; Antonietti, M.; Thomas, A., Porous, Covalent Triazine-Based Frameworks Prepared by lonothermal Synthesis. Angew. Chem. Int. Ed. 2008, 47 (18), 3450-3453.

17. Algara-Siller, G.; Severin, N.; Chong, S. Y.; Björkman, T.; Palgrave, R. G.; Laybourn, A.; Antonietti, M.; Khimyak, Y. Z.; Krasheninnikov, A. V.; Rabe, J. P.; Kaiser, U.; Cooper, A. I.; Thomas, A.; Bojdys, M. J., Triazine-Based Graphitic Carbon Nitride: a Two-Dimensional Semiconductor. Angew. Chem. Int. Ed. 2014, 53 (29), 7450-7455.

18. Guo, X.; Tian, Y.; Zhang, M.; Li, Y.; Wen, R.; Li, X.; Li, X.; Xue, Y.; Ma, L.; Xia, C.; Li, S., Mechanistic Insight into Hydrogen-Bond-Controlled Crystallinity and Adsorption Property of Covalent Organic Frameworks from Flexible Building Blocks. Chem. Mater. 2018, 30 (7), 2299-2308.

19. Liu, M.; Guo, L.; Jin, S.; Tan, B., Covalent triazine frameworks: synthesis and applications. J. Mater. Chem. A 2019, 7 (10), 5153-5172.

20. Noda, Y.; Merschjann, C.; Tarábek, J.; Amsalem, P.; Koch, N.; Bojdys, M. J., Directional Charge Transport in Layered Two-Dimensional Triazine-Based Graphitic Carbon Nitride. Angew. Chem. Int. Ed. 2019, 58 (28), 9394-9398.

21. Tuci, G.; Pilaski, M.; Ba, H.; Rossin, A.; Luconi, L.; Caporali, S.; Pham-Huu, C.; Palkovits, R.; Giambastiani, G., Unraveling Surface Basicity and Bulk Morphology Relationship on Covalent Triazine Frameworks with Unique Catalytic and Gas Adsorption Properties. Adv. Funct. Mater. 2017, 27 (7), 1605672.

22. Zhu, H.; Lin, W.; Li, Q.; Hu, Y.; Guo, S.; Wang, C.; Yan, F., Bipyridinium-Based lonic Covalent Triazine Frameworks for CO2, SO2, and NO Capture. ACS Appl. Mater. Interfaces 2020, 12 (7), 86148621.

23. Lee, Y. J.; Talapaneni, S. N.; Coskun, A., Chemically Activated Covalent Triazine Frameworks with Enhanced Textural Properties for High Capacity Gas Storage. ACS Appl. Mater. Interfaces 2017, 9 (36), 30679-30685.

24. Yang, K.; Yuan, W.; Hua, Z.; Tang, Y.; Yin, F.; Xia, D., Triazine-Based Two-Dimensional Organic Polymer for Selective NO2 Sensing with Excellent Performance. ACS Appl. Mater. Interfaces 2020, 12 (3), 3919-3927.

25. El-Mahdy, A. F. M.; Kuo, C.-H.; Alshehri, A.; Young, C.; Yamauchi, Y.; Kim, J.; Kuo, S.-W., Strategic design of triphenylamine- and triphenyltriazine-based two-dimensional covalent organic frameworks for CO2 uptake and energy storage. J. Mater. Chem. A 2018, 6 (40), 19532-19541.

26. Roeser, J.; Kailasam, K.; Thomas, A., Covalent Triazine Frameworks as Heterogeneous Catalysts for the Synthesis of Cyclic and Linear Carbonates from Carbon Dioxide and Epoxides. ChemSusChem 2012, 5 (9), 1793-1799. 
27. Lan, Z.-A.; Fang, Y.; Zhang, Y.; Wang, X., Photocatalytic Oxygen Evolution from Functional Triazine-Based Polymers with Tunable Band Structures. Angew. Chem. Int. Ed. 2018, 57 (2), $470-474$. 28. Guo, L.; Niu, Y.; Razzaque, S.; Tan, B.; Jin, S., Design of D-A1-A2 Covalent Triazine Frameworks via Copolymerization for Photocatalytic Hydrogen Evolution. ACS Catalysis 2019, 9 (10), 9438-9445.

29. Artz, J., Covalent Triazine-based Frameworks-Tailor-made Catalysts and Catalyst Supports for Molecular and Nanoparticulate Species. ChemCatChem 2018, 10 (8), 1753-1771.

30. Li, Y.; Zheng, S.; Liu, X.; Li, P.; Sun, L.; Yang, R.; Wang, S.; Wu, Z.-S.; Bao, X.; Deng, W.-Q., Conductive Microporous Covalent Triazine-Based Framework for High-Performance Electrochemical Capacitive Energy Storage. Angew. Chem. Int. Ed. 2018, 57 (27), 7992-7996.

31. Vadiyar, M. M.; Liu, X.; Ye, Z., Macromolecular Polyethynylbenzonitrile Precursor-Based Porous Covalent Triazine Frameworks for Superior High-Rate High-Energy Supercapacitors. ACS Appl. Mater. Interfaces 2019, 11 (49), 45805-45817.

32. Hao, L.; Ning, J.; Luo, B.; Wang, B.; Zhang, Y.; Tang, Z.; Yang, J.; Thomas, A.; Zhi, L., Structural Evolution of 2D Microporous Covalent Triazine-Based Framework toward the Study of HighPerformance Supercapacitors. J. Am. Chem. Soc. 2015, 137 (1), 219-225.

33. Tan, C.; Cao, X.; Wu, X.-J.; He, Q.; Yang, J.; Zhang, X.; Chen, J.; Zhao, W.; Han, S.; Nam, G.-H.; Sindoro, M.; Zhang, H., Recent Advances in Ultrathin Two-Dimensional Nanomaterials. Chem. Rev. 2017, 117 (9), 6225-6331.

34. Bojdys, M. J.; Jeromenok, J.; Thomas, A.; Antonietti, M., Rational Extension of the Family of Layered, Covalent, Triazine-Based Frameworks with Regular Porosity. Adv. Mater. 2010, 22 (19), 2202-2205.

35. Wang, K.; Yang, L.-M.; Wang, X.; Guo, L.; Cheng, G.; Zhang, C.; Jin, S.; Tan, B.; Cooper, A., Covalent Triazine Frameworks via a Low-Temperature Polycondensation Approach. Angew. Chem. Int. Ed. 2017, 56 (45), 14149-14153.

36. Ren, S.; Bojdys, M. J.; Dawson, R.; Laybourn, A.; Khimyak, Y. Z.; Adams, D. J.; Cooper, A. I., Porous, Fluorescent, Covalent Triazine-Based Frameworks Via Room-Temperature and MicrowaveAssisted Synthesis. Adv. Mater. 2012, 24 (17), 2357-2361.

37. Troschke, E.; Grätz, S.; Lübken, T.; Borchardt, L., Mechanochemical Friedel-Crafts Alkylation-A Sustainable Pathway Towards Porous Organic Polymers. Angew. Chem. Int. Ed. 2017, 56 (24), 6859-6863.

38. Liu, J.; Zan, W.; Li, K.; Yang, Y.; Bu, F.; Xu, Y., Solution Synthesis of Semiconducting TwoDimensional Polymer via Trimerization of Carbonitrile. J. Am. Chem. Soc. 2017, 139 (34), 1166611669.

39. Liu, J.; Lyu, P.; Zhang, Y.; Nachtigall, P.; Xu, Y., New Layered Triazine Framework/Exfoliated 2D Polymer with Superior Sodium-Storage Properties. Adv. Mater. 2018, 30 (11), 1705401.

40. Yu, S.-Y.; Mahmood, J.; Noh, H.-J.; Seo, J.-M.; Jung, S.-M.; Shin, S.-H.; Im, Y.-K.; Jeon, I.-Y.; Baek, J.-B., Direct Synthesis of a Covalent Triazine-Based Framework from Aromatic Amides. Angew. Chem. Int. Ed. 2018, 57 (28), 8438-8442.

41. Lyu, H.; Diercks, C. S.; Zhu, C.; Yaghi, O. M., Porous Crystalline Olefin-Linked Covalent Organic Frameworks. J. Am. Chem. Soc. 2019, 141 (17), 6848-6852.

42. Acharjya, A.; Pachfule, P.; Roeser, J.; Schmitt, F.-J.; Thomas, A., Vinylene-Linked Covalent Organic Frameworks by Base-Catalyzed Aldol Condensation. Angew. Chem. Int. Ed. 2019, 58 (42), 14865-14870.

43. Jiang, X.; Wang, P.; Zhao, J., 2D covalent triazine framework: a new class of organic photocatalyst for water splitting. J. Mater. Chem. A 2015, 3 (15), 7750-7758.

44. Jin, H.; Guo, C.; Liu, X.; Liu, J.; Vasileff, A.; Jiao, Y.; Zheng, Y.; Qiao, S.-Z., Emerging TwoDimensional Nanomaterials for Electrocatalysis. Chem. Rev. 2018, 118 (13), 6337-6408.

45. Ball, B.; Chakravarty, C.; Sarkar, P., Two-Dimensional Covalent Triazine Framework as a Promising Anode Material for Li-Ion Batteries. J. Phys. Chem. C 2019, 123 (50), 30155-30164.

46. Venkanna, P.; Rajanna, K. C.; Satish Kumar, M.; Bismillah Ansari, M.; Moazzam Ali, M., 2,4,6Trichloro-1,3,5-triazine and N,N'-dimethylformamide as an effective Vilsmeier-Haack reagent for the 
synthesis of 2-chloro-3-formyl quinolines from acetanilides. Tetrahedron Lett. 2015, 56 (37), 51645167.

47. Scott, M. D.; Spedding, H., Vilsmeier adducts of dimethylformamide. J. Chem. Soc. C 1968, (0), 1603-1609.

48. De Luca, L.; Giacomelli, G.; Porcheddu, A., An Efficient Route to Alkyl Chlorides from Alcohols Using the Complex TCT/DMF. Org. Lett. 2002, 4 (4), 553-555.

49. Arenal, R.; March, K.; Ewels, C. P.; Rocquefelte, X.; Kociak, M.; Loiseau, A.; Stéphan, O., Atomic Configuration of Nitrogen-Doped Single-Walled Carbon Nanotubes. Nano Lett. 2014, 14 (10), 5509-5516.

50. Ayala, P.; Arenal, R.; Rümmeli, M.; Rubio, A.; Pichler, T., The doping of carbon nanotubes with nitrogen and their potential applications. Carbon 2010, 48 (3), 575-586.

51. Arenal, R.; De Matteis, L.; Custardoy, L.; Mayoral, A.; Tence, M.; Grazu, V.; De La Fuente, J. M.; Marquina, C.; Ibarra, M. R., Spatially-Resolved EELS Analysis of Antibody Distribution on Biofunctionalized Magnetic Nanoparticles. ACS Nano 2013, 7 (5), 4006-4013.

52. Ayala, P.; Arenal, R.; Loiseau, A.; Rubio, A.; Pichler, T., The physical and chemical properties of heteronanotubes. Rev. Mod. Phys. 2010, 82 (2), 1843-1885.

53. Jia, Y.; Chen, X.; Zhang, G.; Wang, L.; Hu, C.; Sun, X., Topotactic conversion of calcium carbide to highly crystalline few-layer graphene in water. J. Mater. Chem. A 2018, 6 (46), 23638-23643.

54. Casco, M. E.; Kirchhoff, S.; Leistenschneider, D.; Rauche, M.; Brunner, E.; Borchardt, L., Mechanochemical synthesis of $\mathrm{N}$-doped porous carbon at room temperature. Nanoscale 2019, 11 (11), 4712-4718.

55. Chen, X.; Zhang, L.; Zhao, Y.; Wang, X.; Ke, C., Graphene folding on flat substrates. J. Appl. Phys. 2014, 116 (16), 164301.

56. Gholami, M. F.; Severin, N.; Rabe, J. P., Folding of Graphene and Other Two-dimensional Materials. In On Folding: Towards a New Field of Interdisciplinary Research, M. Friedmann, W. S., Ed. transcript: 2016; pp 211-237.

57. Chen, Y.; Wang, X., Template-Free Synthesis of Hollow G-C3N4 Polymer with Vesicle Structure for Enhanced Photocatalytic Water Splitting. J. Phys. Chem. 2018, 122 (7), 3786-3793.

58. Xiang, Z.; Cao, D.; Huang, L.; Shui, J.; Wang, M.; Dai, L., Nitrogen-Doped Holey Graphitic Carbon from 2D Covalent Organic Polymers for Oxygen Reduction. Adv. Mater. 2014, 26 (20), 33153320 .

59. Tahir, M.; Mahmood, N.; Zhu, J.; Mahmood, A.; Butt, F. K.; Rizwan, S.; Aslam, I.; Tanveer, M.; Idrees, F.; Shakir, I.; Cao, C.; Hou, Y., One Dimensional Graphitic Carbon Nitrides as Effective MetalFree Oxygen Reduction Catalysts. Sci. Rep. 2015, 5 (1), 12389.

60. Chen, W.; Huang, L.; Yi, X.; Zheng, A., Lithium doping on 2D squaraine-bridged covalent organic polymers for enhancing adsorption properties: a theoretical study. PCCP 2018, 20 (9), 64876499.

61. Faghani, A.; Donskyi, I. S.; Fardin Gholami, M.; Ziem, B.; Lippitz, A.; Unger, W. E. S.; Böttcher, C.; Rabe, J. P.; Haag, R.; Adeli, M., Controlled Covalent Functionalization of Thermally Reduced Graphene Oxide To Generate Defined Bifunctional 2D Nanomaterials. Angew. Chem. Int. Ed. 2017, 56 (10), 2675-2679.

62. Ferrari, A. C.; Robertson, J.; Ferrari, A. C.; Robertson, J., Raman spectroscopy of amorphous, nanostructured, diamond-like carbon, and nanodiamond. PHILOS T R SOC A 2004, 362 (1824), 24772512.

63. Cataldo, F., The role of Raman spectroscopy in the research on sp-hybridized carbon chains: carbynoid structures polyynes and metal polyynides. J RAMAN SPECTROSC 2008, 39 (2), 169-176.

64. Wang, J.; Zhang, S.; Zhou, J.; Liu, R.; Du, R.; Xu, H.; Liu, Z.; Zhang, J.; Liu, Z., Identifying sp-sp2 carbon materials by Raman and infrared spectroscopies. PCCP 2014, 16 (23), 11303-11309.

65. Namazi, H.; Adeli, M., Solution proprieties of dendritic triazine/poly(ethylene glycol)/dendritic triazine block copolymers. J. Polym. Sci., Part A: Polym. Chem. 2005, 43 (1), 28-41.

66. Agarwal, A.; Chauhan, P. M. S., Convenient Dimethylamino Amination in Heterocycles and Aromatics with Dimethylformamide. Synth. Commun. 2004, 34 (16), 2925-2930. 
67. Heravi, Majid M.; Ghavidel, M.; Mohammadkhani, L., Beyond a solvent: triple roles of dimethylformamide in organic chemistry. RSC Adv. 2018, 8 (49), 27832-27862.

68. Mortensen, J. J.; Hansen, L. B.; Jacobsen, K. W., Real-space grid implementation of the projector augmented wave method. Phys. Rev. B 2005, 71 (3), 035109.

69. Enkovaara, J.; Rostgaard, C.; Mortensen, J. J.; Chen, J.; Dułak, M.; Ferrighi, L.; Gavnholt, J.; Glinsvad, C.; Haikola, V.; Hansen, H. A.; Kristoffersen, H. H.; Kuisma, M.; Larsen, A. H.; Lehtovaara, L.; Ljungberg, M.; Lopez-Acevedo, O.; Moses, P. G.; Ojanen, J.; Olsen, T.; Petzold, V.; Romero, N. A.; Stausholm-Møller, J.; Strange, M.; Tritsaris, G. A.; Vanin, M.; Walter, M.; Hammer, B.; Häkkinen, H.; Madsen, G. K. H.; Nieminen, R. M.; Nørskov, J. K.; Puska, M.; Rantala, T. T.; Schiøtz, J.; Thygesen, K. S.; Jacobsen, K. W., Electronic structure calculations with GPAW: a real-space implementation of the projector augmented-wave method. J. Phys.: Condens. Matter 2010, 22 (25), 253202.

70. Marques, M. A. L.; Oliveira, M. J. T.; Burnus, T., Libxc: A library of exchange and correlation functionals for density functional theory. Comput. Phys. Commun. 2012, 183 (10), 2272-2281.

71. Ghasemi, J.; Shamsipur, M., Fluorimetric Study of Complexation of Alkali and Alkaline Earth Cations with 1,10-Phenanthroline, 2,2'-Bipyridine and 8-Hydroxyquinoline in Nonaqueous Solvents. J. Coord. Chem. 1992, 26 (4), 337-344.

72. Murugavel, R.; Korah, R., Structural Diversity and Supramolecular Aggregation in Calcium, Strontium, and Barium Salicylates Incorporating 1,10-Phenanthroline and 4,4'-Bipyridine: Probing the Softer Side of Group 2 Metal lons with Pyridinic Ligands. Inorg. Chem. 2007, 46 (26), 1104811062.

73. Mitchell, P. R.; Sigel, H., Ternary complexes in solution. Enhanced stability of ternary metal ion/adenosine 5 '-triphosphate complexes. Cooperative effects caused by stacking interactions in complexes containing adenosine triphosphate, phenanthroline, and magnesium, calcium, or zinc ions. J. Am. Chem. Soc. 1978, 100 (5), 1564-1570.

74. Noro, S.-i.; Mizutani, J.; Hijikata, Y.; Matsuda, R.; Sato, H.; Kitagawa, S.; Sugimoto, K.; Inubushi, Y.; Kubo, K.; Nakamura, T., Porous coordination polymers with ubiquitous and biocompatible metals and a neutral bridging ligand. Nat. Commun. 2015, 6 (1), 5851. 


\section{For Table of Contents Only}

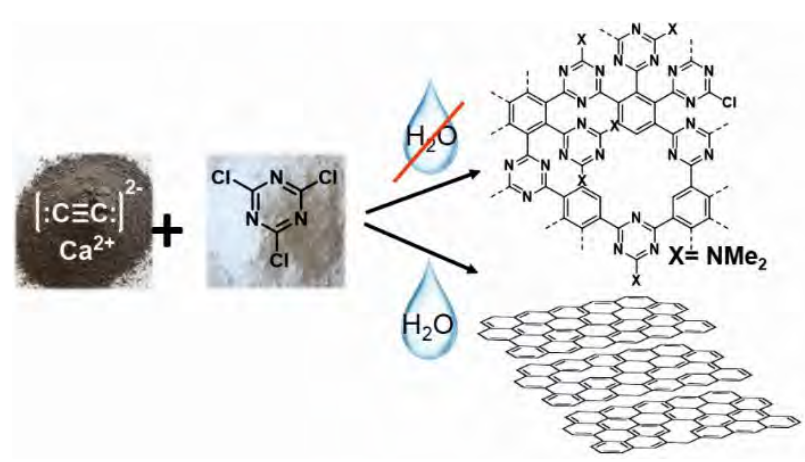

